\title{
Effect of Polarization on the Correlation and Capacity of Indoor MIMO Channels
}

\author{
Hsun-Chang Lo, ${ }^{1}$ Ding-Bing Lin,, ${ }^{2}$ Teng-Chieh Yang, ${ }^{1}$ and Hsueh-Jyh Li ${ }^{3}$ \\ ${ }^{1}$ Information and Communications Research Laboratories, Industrial Technology Research Institute, Hsinchu 31040, Taiwan \\ ${ }^{2}$ Department of Electronic Engineering, National Taipei University of Technology, Taipei 10608, Taiwan \\ ${ }^{3}$ Graduate Institute of Communication Engineering, National Taiwan University, Taipei 10617, Taiwan \\ Correspondence should be addressed to Hsun-Chang Lo, shunchanglo@itri.org.tw
}

Received 17 June 2011; Revised 30 August 2011; Accepted 31 August 2011

Academic Editor: Yan Zhang

Copyright (๑) 2012 Hsun-Chang Lo et al. This is an open access article distributed under the Creative Commons Attribution License, which permits unrestricted use, distribution, and reproduction in any medium, provided the original work is properly cited.

\begin{abstract}
We describe a simple multiple-input/multiple-output (MIMO) channel measurement system for acquiring indoor MIMO channel responses. Four configurations of the polarization diversity antenna, referred to as VVV, YYH, YVY and VHH, were studied in terms of the capacity of indoor MIMO systems. Measurements were taken for a $3 \times 3$ MIMO system in the $2.4 \mathrm{GHz}$ band. In addition, the channel capacity, singular-value decomposition, and correlation coefficient were used to explain the effects of various polarization schemes on MIMO fading channels. We also propose an analysis method for polarization channel capacity; this method includes the normalization of the received power and polarization effect for different polarization schemes. The validation of the model is based upon data collected in both light-of-sight (LOS) and non-light-of-sight (NLOS) environments. From the numerical simulation results, the proposed analysis method was close to measurements made in an indoor environment.
\end{abstract}

\section{Introduction}

The multiple-input/multiple-output (MIMO) system proposed by Foschini [1] is a remarkable structure that achieves large capacity through parallel channeling. However, the capacity of an MIMO system is highly dependent on the correlation properties of the channels. It is well known that because of the high isolation of orthogonal polarizations, using a cross-polarization antenna scheme symmetrically at both transmitting and receiving ends can provide a higher channel capacity than a conventional antenna polarization scheme. Moreover, part of the existing infrastructure uses cross-polarized antenna elements, which could be used to support both polarization diversity and polarization multiplexing [2]. The channel capacity for MIMO systems has been investigated theoretically [3-5]. In addition, polarization diversity that employs both vertical and horizontal polarizations can make the fading correlation between copolarized and cross-polarized channels sufficiently low, regardless of the antenna spacing [6-8].

Recently, many researchers have examined multiple polarizations for an MIMO antenna system. Andrews et al.
[9] discussed a wireless MIMO link that provides six independent signals with three electric dipoles and three magnetic dipoles to take full rank of the channel. They assumed an antenna model with ideal polarizations and a rich scattering environment and confirmed their analysis using three orthogonal electric dipoles. $\mathrm{Li}$ and $\mathrm{Yu}$ [10] compared the MIMO correlation coefficient properties and channel capacities, and displayed different polarization combinations are an efficient way for enhancing channel capacity. Svantesson [11] studied the effect of multipath angular spread and antenna radiation patterns on the channel capacity and showed that the capacity increase is due to a combination of polarization and pattern diversity. Weichselberger and Özcelik [12]. introduced a formulation for narrowband MIMO channel model characterized by eigenvectors of covariance matrices and coupling matrices to fulfill the deficiencies. The structure of formulation resembles virtual channel representation [13], in which steering vectors and virtual channel representation matrix are involved.

On the other hand, one way of reducing the level of correlation between antenna elements is to provide sufficient interelement spacing. However, for the practical application 
of MIMO systems to a wireless local area network, the spacing between adjacent antenna elements cannot be too large. Given this constraint, the most probable solution is a dense MIMO array with nonnegligible correlation. In addition, the use of polarization diversity may be a solution for obtaining a more compact antenna array layout since another diversity dimension can be provided to the MIMO radio channel. Therefore, modeling the polarization diversity technique is an interesting topic of study in MIMO radio channels, and it necessitates the construction of realistic MIMO radio channel models featuring both space and polarization diversity [14-16].

Dissimilar channel environments and different incoming wave angular distributions result in a distinct spatial correlation among antenna array elements, which are the main parameters that affect MIMO channel characteristics [17]. To investigate the system performance and correlation properties, we use various antenna polarization combinations in different environments for the same antenna element spacing.

The validation of the model is supported by measurement results. Using two measurement setups having several transmitting and receiving elements, four polarization schemes have been investigated in several different LOS and NLOS rooms as indoor environments. The parameters of the MIMO model are extracted from the measurement data and fed to the model to compare simulation results with the measurement results.

In this paper, we propose an analysis method, which utilizes a correlation coefficient for both transmission and reception and the average receiving power, to calculate the MIMO channel capacity in polarization systems. In addition, we derive the optimal received-power matrix for both additive white Gaussian noise (AWGN) and multipath fading channels. Furthermore, measurement results show that the proposed analysis method outperforms traditional method in polarization systems.

The remainder of this paper is organized as follows. In Section 2, the experiments on the channel measurement setup and antenna configuration are described in detail. In Section 3, we discuss the concepts of the MIMO system, including the singular-value decomposition (SVD) method for finding channel gain, subchannel correlations, and channel capacity. The normalization method for comparing the effect of various polarization schemes is mentioned in this section. In Section 4, the channel capacity and correlation coefficient of the measured channel are calculated, and some meaningful results are revealed. Finally, we present conclusions in Section 5.

\section{Measurement Setup}

MIMO measurements with an $M \times N$ setup, where $N$ and $M$ are the numbers of elements at the transmitter (Tx) and receiver $(\mathrm{Rx})$, respectively, are conducted with $M=N=3$. A simplified sketch of the MIMO measurement setup is shown in Figure 1.

To measure the response of the single-input/singleoutput (SISO) channel, a two-port vector network analyzer

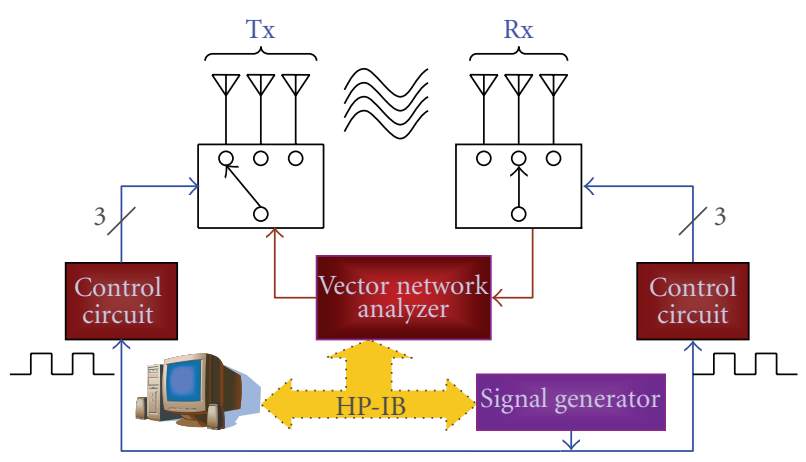

FIGURE 1: MIMO measurement system.

TAble 1: Parameters of measurement.

\begin{tabular}{lc}
\hline Central frequency & $2.4 \mathrm{GHz}$ \\
\hline Frequency span & $500 \mathrm{MHz}$ \\
Number of frequency points & 801 \\
Antenna type & $2.4 \mathrm{GHz}$ omniantenna \\
No. of transmit antennas & 3 \\
No. of receive antennas & 3 \\
\hline
\end{tabular}

is used. Both Tx and Rx are attached to a 1-to-3 switch with switching times of $15 \mathrm{~s}$ and $5 \mathrm{~s}$ for $\mathrm{Tx}$ and $\mathrm{Rx}$, respectively. Furthermore, a signal generator is required to control the status of the switches. A personal computer is used to acquire the measured data and to remotely control the signal generator so that the correct clock signal is sent through the general-purpose interface bus. During the measurement, one of the transmitting antennas is switched on, and three receiving antennas are activated one after the other. Next, the second transmitting antenna is switched on, and the control circuit at the receiver activates the receiving antennas successively. The process is repeated for all the transmitting antennas. We assume that the indoor channel is quasistatic so that we can measure each radio link by controlling the switching circuits. The measuring time for each link is $5 \mathrm{~s}$, so it takes about $45 \mathrm{~s}$ to complete a $3 \times 3$ MIMO channel matrix measurement. The parameters of our measurement are listed in Table 1.

2.1. Measurement Environment. The measurement sites are located in Electrical Engineering Building II at the National Taiwan University campus. Two dissimilar environments are selected for their different characteristics. Figure 2 shows the equipment and setting for indoor channel measurements. The size of the room is about $8 \mathrm{~m} \times 8 \mathrm{~m}$. The transmitting antenna array is fixed at the center of the classroom, and the receiver is placed at eight locations. These locations lie on the circumference of a circle with a radius of $3 \mathrm{~m}$ that is centered at the center of the room; this means that all eight locations are equidistant from the transmitter. Each receiving antenna array is moved around a $1-\mathrm{m}^{2}$ area to obtain channel responses for sixteen positions. As a result, we obtain a total of 128 MIMO channel matrices at the end of the experiment. Figure 3 shows the placement of equipment in two rooms on 


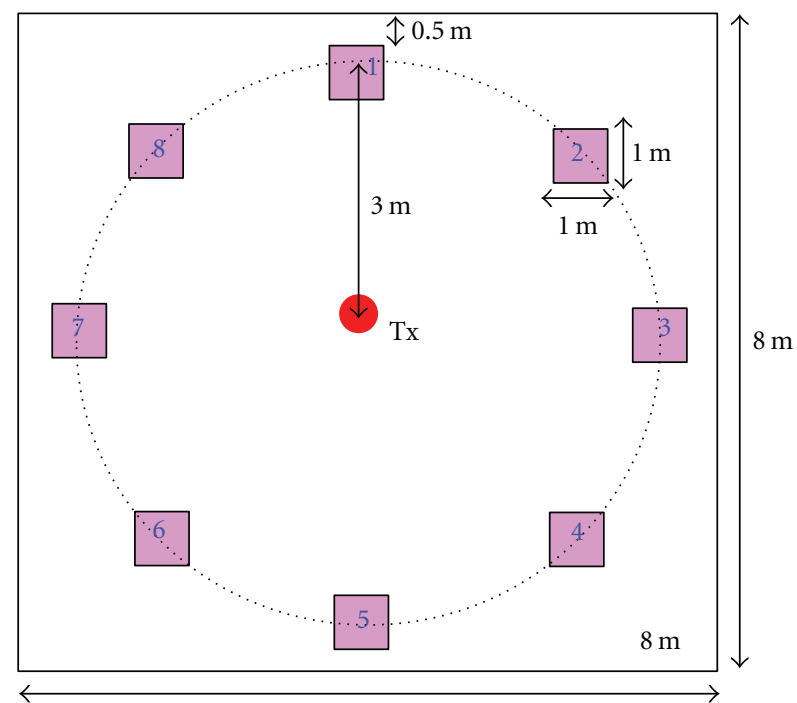

FIgURE 2: The experimental layout for indoor LOS environment.

the same floor for the NLOS environment. There is a concrete wall with a thickness of $10 \mathrm{~cm}$ between the two rooms. In Figure 3, the locations of the receiving and transmitting antenna arrays are denoted as " $\mathrm{X}$ " and "•", respectively. The height of the antenna arrays at $\mathrm{Tx}$ and $\mathrm{Rx}$ is $1.2 \mathrm{~m}$. The measurements are made in the absence of bodily movement to investigate time-stationary picocell environments.

For both environments, a large number of receiving locations are used to gather more statistical information for the environment. In addition, to remove the effect of attenuation or loss resulting from equipment, we calibrate the data by dividing the measured frequency response by that measured for 1-m Tx-Rx separation.

2.2. Antenna Configuration. In this study, we investigate different polarization configurations: vertical $(\mathrm{V})$, horizontal $(\mathrm{H})$, and slanting $(\mathrm{Y})$ polarizations (angle of slant $= \pm 45^{\circ}$ ). The relative orientations of the transmitting antenna array and receiving antenna array are shown in Figure 4. Whenever the receiving antenna array is moved, the same relative orientation between the transmitting antenna array and the receiving antenna is maintained for the same polarization type. Figure 5 shows photos of the four different polarization combinations, in which each antenna has an omnidirectional pattern and a 5-dBi antenna gain. At both transmitting and receiving ends, the adjacent antennas are spaced half wavelengths apart. Transmission is carried out at $2.4 \mathrm{GHz}$ center frequency with a frequency span of $500 \mathrm{MHz}$ over 801 individual frequencies (points).

\section{Narrowband MIMO Channel Model Analysis}

In this section, we analyze the measurement results obtained for the MIMO radio channels used in narrowband systems. Although our measurement system is wideband, we only use data in the frequency range $2.35-2.45 \mathrm{GHz}$, corresponding to 161 frequency points in the central region, because the antenna possesses a narrowband operational bandwidth. Thus, our discussion deals with narrowband analysis. Moreover, to analyze the capacity and eigenvalue of the measured data, we first need to normalize the data. For each location, let $H_{f}^{k}$ denote the channel matrix measured at the $k$ th position $(k=1-16)$ and $f$ th frequency point.

3.1. Normalization for Different Polarizations. We discuss the relationship between polarization and channel capacity. The method for normalizing the VVV configuration is defined as follows:

$$
\begin{gathered}
\frac{1}{F \times K \times M \times N} \sum_{f} \sum_{k}\left\|H_{f}^{k}\right\|_{F}^{2}=E, \\
\|H\|_{F}^{2}=\sum_{i=1}^{M} \sum_{j=1}^{N}\left|h_{i j}\right|^{2}, \\
\mathbf{H}=\left[\begin{array}{cccc}
h_{11} & h_{12} & \cdots & h_{1 N} \\
h_{21} & h_{22} & \cdots & h_{2 N} \\
\vdots & \vdots & \ddots & \vdots \\
h_{M 1} & h_{M 2} & \cdots & h_{M N}
\end{array}\right], \\
\hat{H}_{f}^{k}=\frac{H_{f}^{k}}{\sqrt{E}},
\end{gathered}
$$

where $\mathbf{H}$ is an $M \times N$ channel matrix at one position and one frequency point. $\|H\|_{F}^{2}$ is the square of the Frobenius norm of $\mathbf{H}$. Moreover, $k$ denotes the $k$ th position (where the total number of positions in one location is denoted as $K$ ) in each location and $f$ denotes the $f$ th frequency point (where the total number of frequency points is denoted as $F$ ).

The normalization method is different for the YVY case. We use the VVV case for the same location as the baseline. We first calculate the mean power of all antenna elements in the VVV case over all the frequency points and positions in the same location. We then normalize all the YVY channel matrices by the square root of the mean power for the VVV case:

$$
\begin{gathered}
\frac{1}{F \times K \times M \times N} \sum_{f} \sum_{k}\left\|\left.H_{f}^{k}\right|_{\mathrm{VVV}}\right\|_{F}^{2}=E, \\
\left.\hat{H}_{f}^{k}\right|_{\mathrm{YVY}}=\frac{\left.H_{f}^{K}\right|_{\mathrm{YVY}}}{\sqrt{E}},
\end{gathered}
$$

where $\left.H_{f}^{k}\right|_{\mathrm{VVV}}$ and $\left.H_{f}^{k}\right|_{\mathrm{YVY}}$ denote the channel matrices at the $k$ th position and $f$ th frequency point for the VVV case and YVY case, respectively. The normalization methods for the other cases are the same as the method used for YVY normalization. 


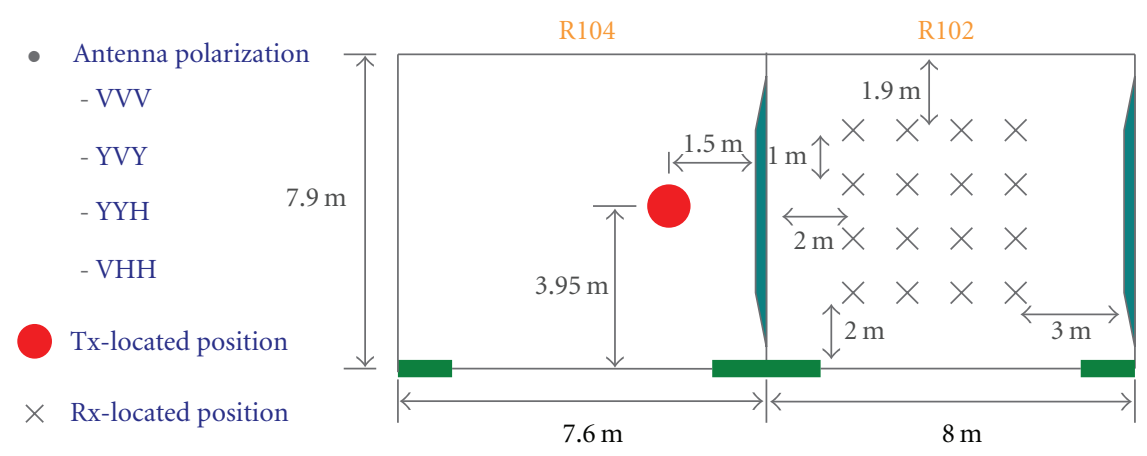

FIgURE 3: The experimental layout for indoor NLOS environment.

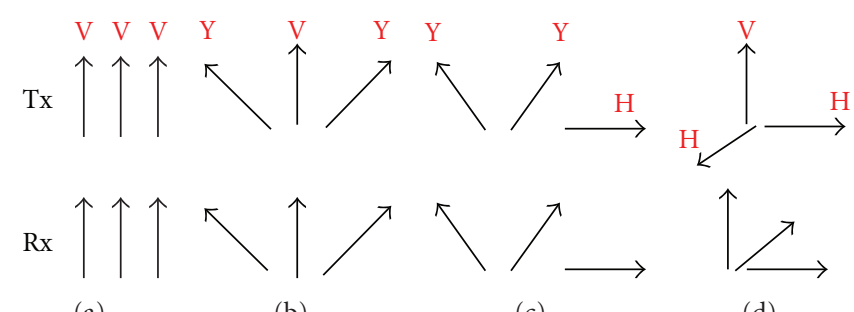

(a)

(b)

(c)

(d)

Figure 4: Four antenna configurations which are denoted as (a) VVV, (b) YVY, (c) YYH, and (d) VHH.

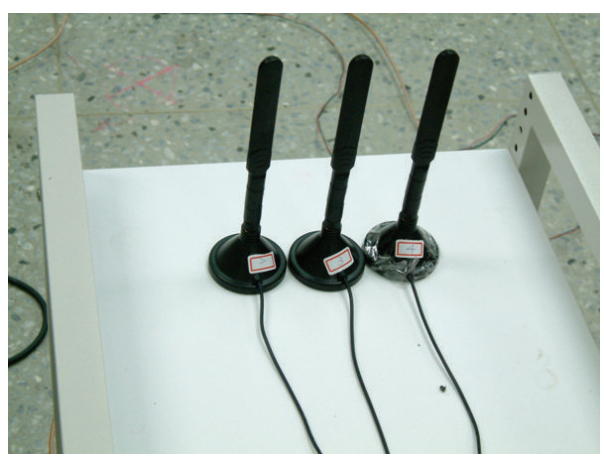

(a)

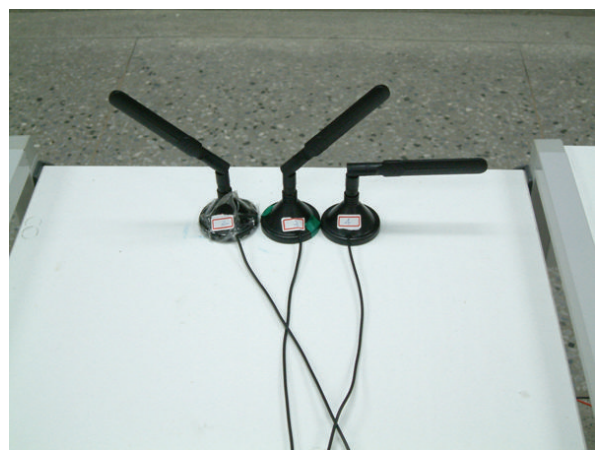

(c)

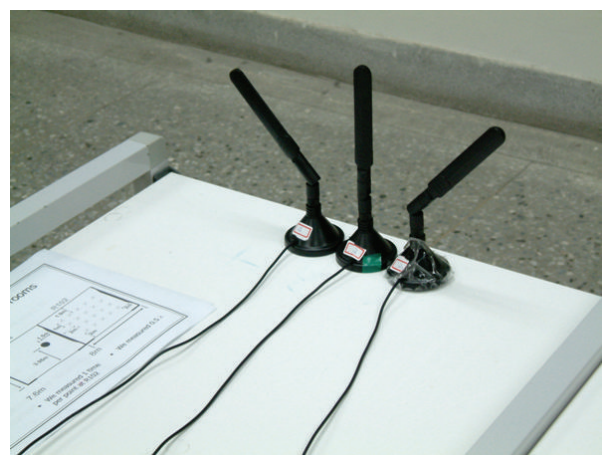

(b)

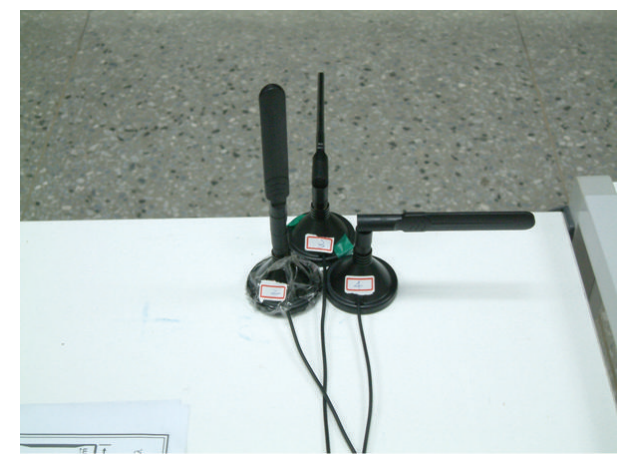

(d)

FIgure 5: Photos of the four different polarization combinations denoted as (a) VVV, (b) YVY, (c) YYH, and (d) VHH in practical measurement environment. 
3.2. Eigenvalue and Capacity for Equal Power. In analyzing the capacity of an MIMO system, $N$ transmitting elements and $M$ receiving elements are assumed, and the received signal is expressed as

$$
\mathbf{y}=\mathbf{H s}+\mathbf{n}
$$

where $\mathbf{H}$ is an $M \times N$ channel matrix, $\mathbf{s}$ is the transmitted signal, and $\mathbf{n}$ is the AWGN.

For a fixed channel realization, the channel capacity has the following constraints: (1) the transmitter has no channel state information and (2) the transmitted power is equally allocated to each transmitting element. Therefore, the capacity can be expressed as [1]

$$
C=\log _{2} \operatorname{det}\left(\mathbf{I}_{M}+\frac{\rho}{N} \mathbf{H H}^{H}\right) \quad \text { bits/s/Hz, }
$$

where $\operatorname{det}(\cdot)$ denotes the determinant of a matrix, and $(\cdot)^{H}$ denotes a Hermitian transpose. $\mathbf{I}_{M}$ is an $M \times M$ identity matrix and $\rho$ is the average received signal-to-noise ratio.

To investigate the characteristics of $\mathbf{H}$, we can perform an SVD of $\mathbf{H}$ to diagonalize $\mathbf{H}$ and determine the eigenvalues. The SVD expansion of any matrix $\mathbf{H}(M \times N)$ is written as [18]

$$
\mathbf{H}=\mathbf{U} \boldsymbol{\Lambda} \mathbf{V}^{H},
$$

where $\mathbf{U}(M \times M)$ and $\mathbf{V}(N \times N)$ are unitary matrices, which means that $\mathbf{U} \mathbf{U}^{H}=\mathbf{V} \mathbf{V}^{H}=\mathbf{I} . \boldsymbol{\Lambda}(M \times N)$ is nonnegative and diagonal with entries specified by

$$
\boldsymbol{\Lambda}=\operatorname{diag}\left(\sqrt{\lambda_{1}}, \sqrt{\lambda_{2}}, \ldots, \sqrt{\lambda_{q}}, 0, \ldots, 0\right),
$$

where $\operatorname{diag}(\boldsymbol{\Lambda})$ is a vector consisting of the diagonal elements of $\Lambda ; \lambda_{1}, \lambda_{2}, \ldots, \lambda_{q}$ are the nonzero eigenvalues of $\mathbf{W} ; q=$ $\min (M, N)$.

The columns of $\mathbf{U}$ and $\mathbf{V}$ are the eigenvectors of $\mathbf{H H}^{H}$ and $\mathbf{H}^{H} \mathbf{H}$ [19], respectively. The SVD (8) shows that the channel matrix $\mathbf{H}$ can be diagonalized to a number of independent orthogonal subchannels, where the power gain of the $i$ th channel is $\lambda_{i}$ [20],

$$
\mathbf{W}= \begin{cases}\mathbf{H H}^{H}, & M \leq N, \\ \mathbf{H}^{H} \mathbf{H}, & N<M .\end{cases}
$$

Thus, (7) can be rewritten as [20]

$$
C_{\mathrm{EP}}=\sum_{i=1}^{q} \log _{2}\left(1+\frac{\rho}{N} \lambda_{i}\right) \quad \mathrm{bps} / \mathrm{Hz},
$$

where $\lambda_{1}, \lambda_{2}, \ldots, \lambda_{q}$ are nonzero eigenvalues of $\mathbf{W}$ in (10).

Therefore, the channel capacity is affected not only by the maximal value of $\lambda_{\mathrm{i}}$ but also by the minimal value of $\lambda_{\mathrm{i}}$. We can define the condition number as

$$
\text { condition number }=\frac{\lambda_{\max }}{\lambda_{\min }} .
$$

If a channel has a low condition number, then its correlation is low, its diversity is high, and thus its capacity is high. The channel is then said to be "well conditioned." Otherwise, the channel is referred to as being "ill conditioned" [21].
3.3. Subchannel Correlation. We modify the method of analysis of channel capacity for different polarization schemes. A different polarization configuration has a different received power owing to cross-polarization. Therefore, we consider the effect of the cross-polarization ratio (XPR) in different polarization schemes. Moreover, the use of the XPR for polarization antenna systems has been previously studied.

The XPR is defined as the ratio of the copolarized average received power to the cross-polarized average received power. The XPR has been used in the capacity analysis of different polarization combinations [22].

To obtain XPRs for different polarization cases, we normalize the VVV scheme so that the average normalized power matrix is equal to one. The average received power matrix $\mathbf{P}$ is defined as

$$
\begin{gathered}
P_{i, j}=\frac{1}{K} \sum_{k} \frac{1}{F} \sum_{f}\left|h_{i, j}\right|_{k, f}^{2}, \\
\mathbf{P}_{O}=\left[\begin{array}{cccc}
P_{1,1} & P_{1,2} & \cdots & P_{1, N} \\
P_{2,1} & P_{2,2} & \cdots & P_{2, N} \\
\vdots & \vdots & \ddots & \vdots \\
P_{M, 1} & P_{M, 2} & \cdots & P_{M, N}
\end{array}\right] \in \mathrm{R}^{M \times N} .
\end{gathered}
$$

From (13), we obtain the matrix $\mathbf{P} \in R^{M \times N}$ for different polarization schemes. Next, we obtain $\mathbf{P}_{O}$ as the normalized power matrix and use the VVV case in the same location as the baseline. Other polarization schemes refer to the VVV scheme. Finally, the proposed MIMO channel matrix is written as

$$
\begin{gathered}
\mathbf{H}=\mathbf{R}_{H, \mathrm{Rx}}^{1 / 2} \mathbf{G P}_{O}{ }^{1 / 2}\left[\mathbf{R}_{H, \mathrm{Tx}}^{1 / 2}\right]^{T}, \\
\mathfrak{R}_{H}^{\mathrm{Tx}}=\left[\begin{array}{cccc}
\rho_{11}^{\mathrm{Tx}} & \rho_{12}^{\mathrm{Tx}} & \cdots & \rho_{1 N}^{\mathrm{Tx}} \\
\rho_{21}^{\mathrm{Tx}} & \rho_{22}^{\mathrm{Tx}} & \cdots & \rho_{2 N}^{\mathrm{Tx}} \\
\vdots & \vdots & \ddots & \vdots \\
\rho_{N 1}^{\mathrm{Tx}} & \rho_{N 2}^{\mathrm{Tx}} & \cdots & \rho_{N N}^{\mathrm{Tx}}
\end{array}\right]_{N \times N} \\
\mathfrak{R}_{H}^{\mathrm{Rx}}=\left[\begin{array}{cccc}
\rho_{11}^{\mathrm{Rx}} & \rho_{12}^{\mathrm{Rx}} & \cdots & \rho_{1 M}^{\mathrm{Rx}} \\
\rho_{21}^{\mathrm{Rx}} & \rho_{22}^{\mathrm{Rx}} & \cdots & \rho_{2 M}^{\mathrm{Rx}} \\
\vdots & \vdots & \ddots & \vdots \\
\rho_{M 1}^{\mathrm{Rx}} & \rho_{M 2}^{\mathrm{Rx}} & \cdots & \rho_{M M}^{\mathrm{Rx}}
\end{array}\right]_{M \times M},
\end{gathered}
$$

where $\mathbf{G}$ is an $M \times M$ matrix containing independent and identically distributed $\mathbf{C N}(0,1)$ elements, and $\mathbf{R}_{H}^{\mathrm{Tx}}$ and $\mathbf{R}_{H}^{\mathrm{Rx}}$ are transmitting and receiving correlation matrices respectively. Then, $\rho_{i j}^{\mathrm{Tx}}$ ( or $\rho_{i j}^{\mathrm{Rx}}$ ) is the correlation coefficient of the $i$ th and $j$ th transmitting (or receiving) antennas. 


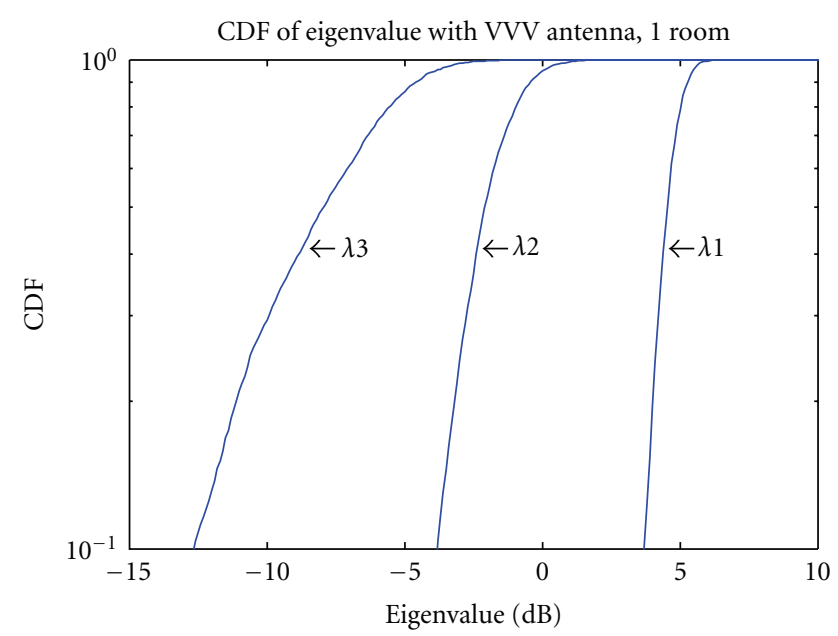

(a)

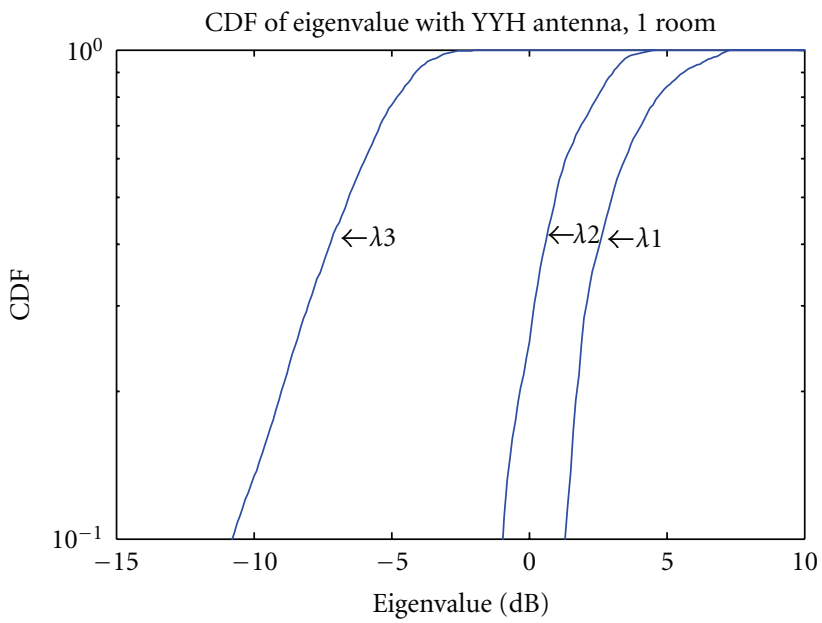

(c)

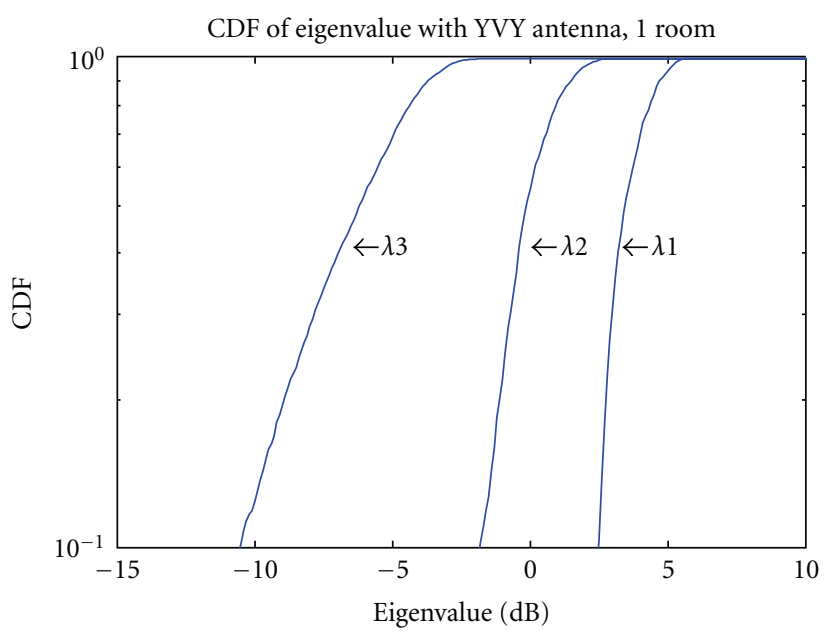

(b)

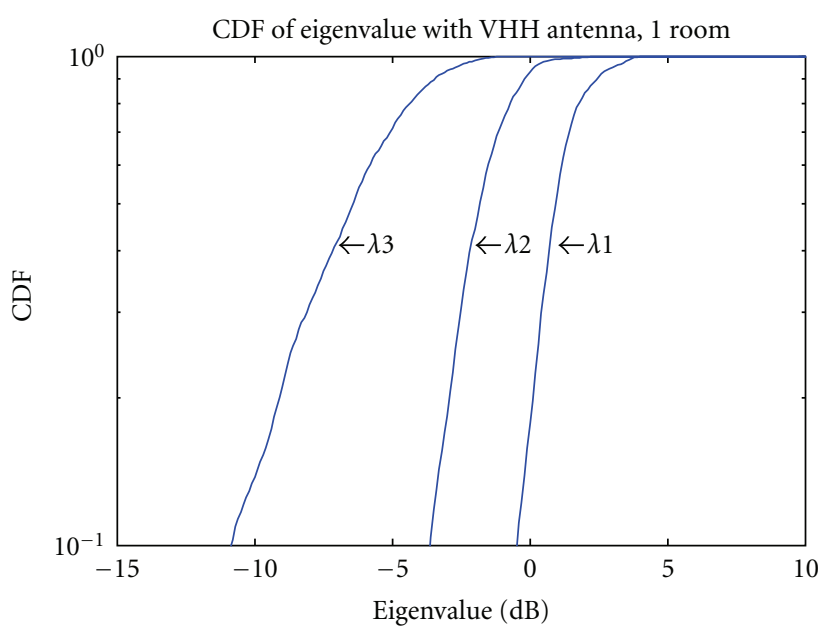

(d)

Figure 6: The plots of CDF versus eigenvalues when the polarization system employing (a) VVV, (b) YVY, (c) YYH, and (d) VHH cases in LOS indoor environment.

For a measured MIMO channel matrix $\mathbf{H} \in \mathrm{C}^{M \times N}$ as denoted in (3), we can thus define the transmitter and receiver correlation coefficients as

$$
\begin{aligned}
& \rho_{n_{1} h_{2}}^{\mathrm{Tx}}(k, f)=\left\langle h_{m n_{1}}, h_{m n_{2}}\right\rangle=\frac{\sum_{m} h_{m, n_{1}} h_{m, n_{2}}^{*}}{\sqrt{\sum_{m}\left|h_{m, n_{1}}\right|^{2} \sum_{m}\left|h_{m, n_{2}}\right|^{2}}}, \\
& \rho_{m_{1} m_{2}}^{\mathrm{Rx}}(k, f)=\left\langle h_{m_{1} n}, h_{m_{2} n}\right\rangle=\frac{\sum_{n} h_{m_{1}, n} h_{m_{2}, n}^{*}}{\sqrt{\sum_{n}\left|h_{m_{1}, n}\right|^{2} \sum_{n}\left|h_{m_{2}, n}\right|^{2}}} .
\end{aligned}
$$

Subsequently, the correlation coefficients are calculated as follows:

$$
\rho_{n_{1}, n_{2}}^{\mathrm{Tx}}(f)=\frac{1}{K} \sum_{k} \rho_{n_{1}, n_{2}}^{\mathrm{Tx}}(k, f) \Longrightarrow \rho_{n_{1}, n_{2}}^{\mathrm{Tx}}=\frac{1}{F} \sum_{f}\left|\rho_{n_{1}, n_{2}}^{\mathrm{Tx}}\right|,
$$

$$
\rho_{m_{1}, m_{2}}^{\mathrm{Rx}}(f)=\frac{1}{K} \sum_{k} \rho_{m_{1}, m_{2}}^{\mathrm{Rx}}(k, f) \Longrightarrow \rho_{m_{1}, m_{2}}^{\mathrm{Rx}}=\frac{1}{F} \sum_{f}\left|\rho_{m_{1}, m_{2}}^{\mathrm{Rx}}\right| .
$$

\section{Measurement Results}

Our experiment focuses on indoor measurements. We intend to gain a deeper understanding of the effects of different polarizations from our experimental results.

The first and the second MIMO scenario performances are illustrated in terms of the cumulative distribution functions (CDFs) of their eigenvalues, as shown in Figures 6 and 7 , in which three subchannel gains are plotted as straight lines. We find that regardless of whether the LOS or NLOS environment is used, the largest eigenvalue $\left(\lambda_{1}\right)$ in the VHH schemes is smaller than $\lambda_{1}$ of other polarization schemes owing to the significantly lower channel gain. Moreover, $\lambda_{1}$ is higher for the VVV scheme than for the YVY, YYH, and VHH schemes; thus, the channel capacity of the VHH 


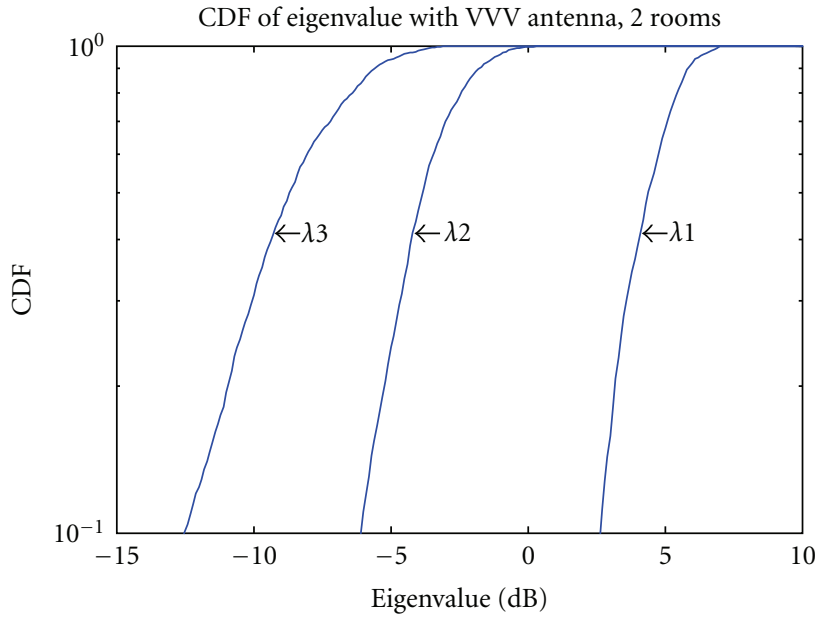

(a)

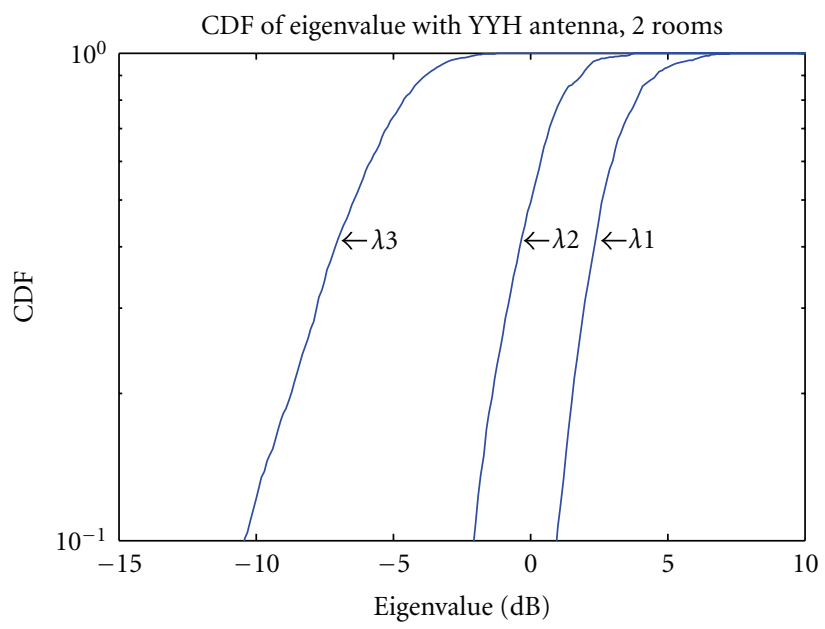

(c)

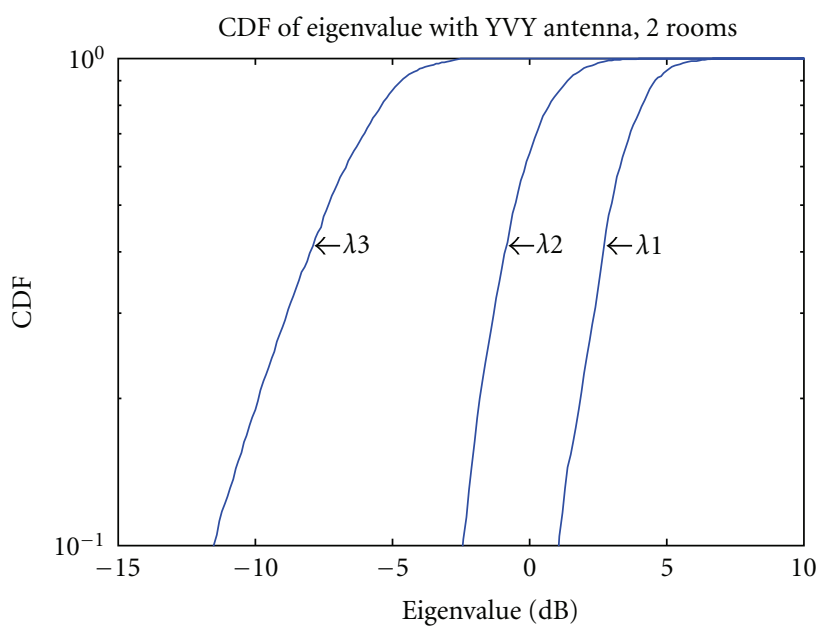

(b)

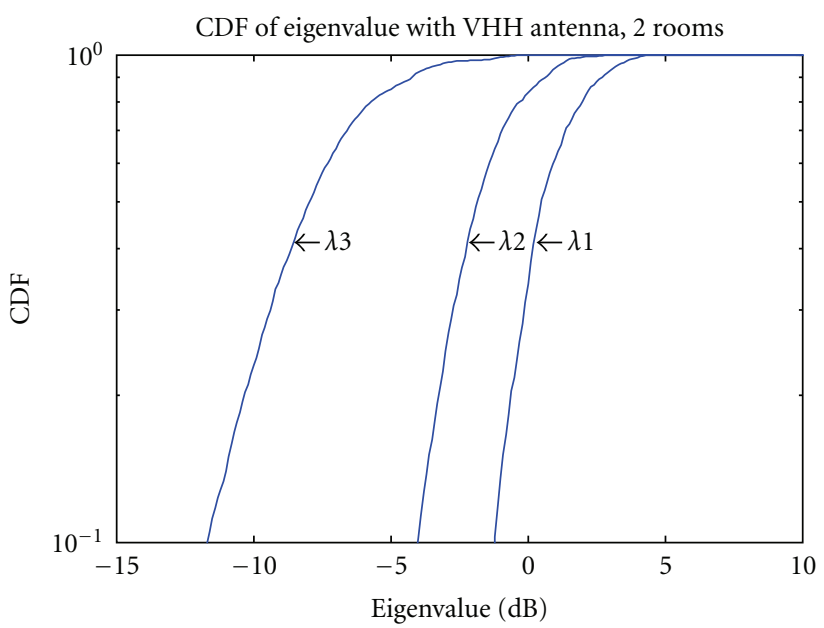

(d)

Figure 7: The plots of CDF versus eigenvalues when the polarization system employing (a) VVV (b) YVY (c) YYH (d) VHH cases in NLOS indoor environment.

combination in indoor environments is the smallest among the capacities of all the schemes. However, all the eigenvalues are closer to each other in the VHH case than in the other polarization cases. Hence, the VHH channel is the most well conditioned.

Let us consider the channel correlation matrices listed in Tables 2 and 3. We observe that the VVV copolarized cases have greater correlation coefficients than the other polarization cases. In addition, if the polarization between the transmitting antenna and receiving antenna is orthogonal, then the correlation coefficient is the lowest among all the polarized pairs under the same measurement environment. From this viewpoint, we study the YYH polarization scheme. The correlation coefficient $\rho_{12}\left(\rho_{21}\right)$ is smaller than $\rho_{13}\left(\rho_{31}\right)$ and $\rho_{23}\left(\rho_{32}\right)$ owing to orthogonal polarization transmission. The same relationship holds for the YVY and VHH polarization schemes. Consequently, the correlation coefficients of the nondiagonal matrix elements are lower; the channel of the MIMO systems is more uncorrelated; the channel has a higher capacity.

We also examine the correlation for direct path power which defines the first path in time domain among the subchannels in the four polarization schemes. As shown in Tables 4 and 5, if the polarization between transmitting antenna and receiving antenna is orthogonal, then the correlation coefficient of the direct path power corresponds to the lowest subchannel gain among all the polarized pairs. All matrix components of VVV are 1 because of the direct path powers are close to each other. In explaining the effects of polarization combinations, we use the notation $\mathrm{P}-\mathrm{Q}$, where $\mathrm{P}$ and $\mathrm{Q}$ denote the transmitting antenna and receiving antenna, respectively. Considering the normalized power matrix $\left(\mathbf{P}_{N}\right)$ in Tables 6 and 7 , we note that the V-V copolarized case and the $\mathrm{Y}-\mathrm{Y}$ ("V"- "H") cross-polarized case are very 
TABLE 2: Correlation matrix for different polarization schemes in LOS environment.

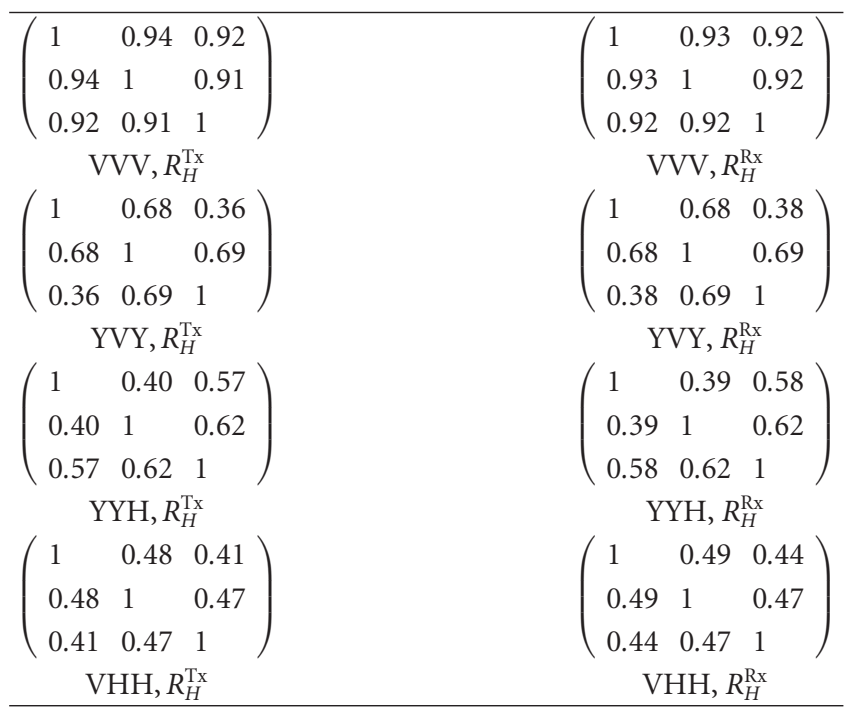

TABLE 3: Correlation matrix for different polarization schemes in NLOS environment.

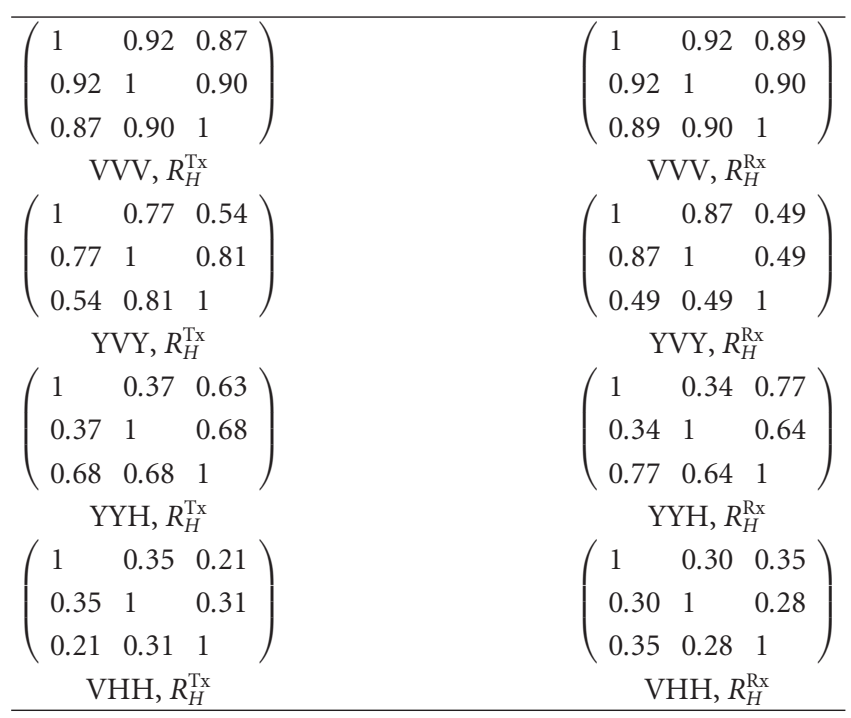

different. The YVY cross-polarized combination shows three subchannels with considerably high gain and remaining subchannels with a lower gain; in contrast, the VVV copolarized combination shows high gains for all the subchannels, with the gains being close to each other, although the $\mathrm{VHH}$ scheme provides good isolation as the antenna elements are orthogonal for the transmission and reception pairs. Nevertheless, as we observe normalized received power matrices, it is almost the loss of all the subchannel gains. This phenomenon shows the benefit of adopting orthogonal polarizations. Table 8 lists the gains of different polarization schemes; these gains help to clarify the above phenomenon. As a result, in using orthogonal polarization, we lose part of the channel gain but achieve greater isolation. The same results are found for the YYH scheme.
TABLE 4: Correlation matrix of direct path power analysis for different polarization schemes in LOS environment.

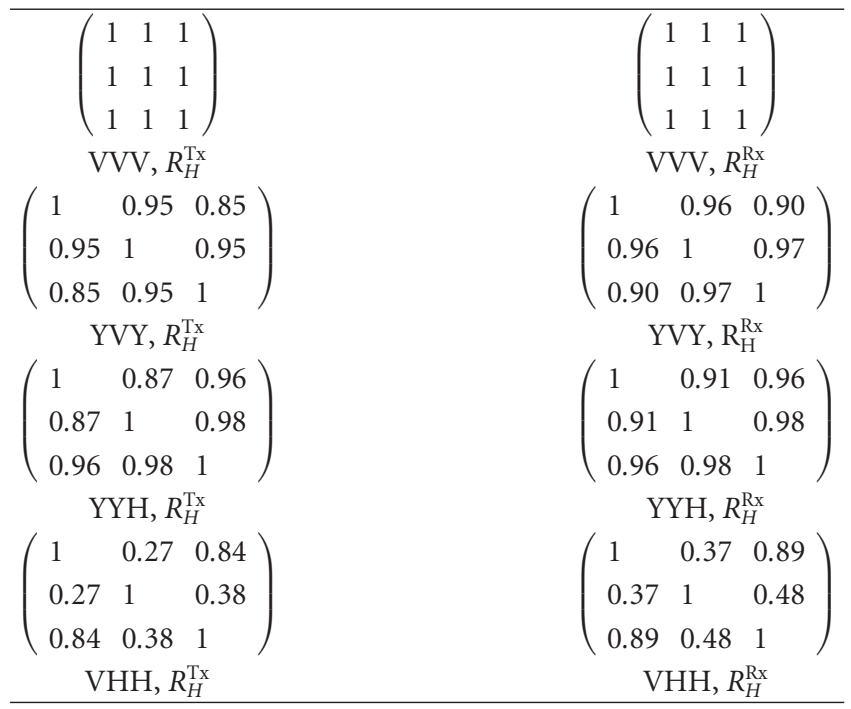

TABLE 5: Correlation matrix of direct path power analysis for different polarization schemes in NLOS environment.

\begin{tabular}{|c|c|}
\hline$\left(\begin{array}{lll}1 & 1 & 1\end{array}\right)$ & $\left(\begin{array}{lll}1 & 1 & 1\end{array}\right)$ \\
\hline \begin{tabular}{lll|l}
1 & 1 & 1 \\
1 & 1 & 1
\end{tabular} & $\begin{array}{lll}1 & 1 & 1 \\
1 & 1\end{array}$ \\
\hline$\left|\begin{array}{lll}1 & 1 & 1\end{array}\right|$ & \begin{tabular}{lll|}
1 & 1 & 1
\end{tabular} \\
\hline$\left(\begin{array}{lll}1 & 1 & 1\end{array}\right)$ & $\left(\begin{array}{lll}1 & 1 & 1\end{array}\right)$ \\
\hline $\mathrm{VVV}, R_{H}^{\mathrm{Tx}}$ & $\mathrm{VVV}, R_{H}^{\mathrm{Rx}}$ \\
\hline$\left(\begin{array}{lll}1 & 0.93 & 0.82\end{array}\right)$ & $\left.\begin{array}{ll}0.91 & 0.85\end{array}\right)$ \\
\hline $\begin{array}{lll}0.93 & 1 & 0.95\end{array}$ & $\begin{array}{lll}0.91 & 1 & 0.93\end{array}$ \\
\hline $\begin{array}{lll}0.82 & 0.95 & 1\end{array}$ & $\begin{array}{lll}0.85 & 0.93 & 1\end{array}$ \\
\hline $\mathrm{YVY}, R_{H}^{\mathrm{Tx}}$ & $\mathrm{YVY}, R_{H}^{\mathrm{Rx}}$ \\
\hline$\left(\begin{array}{lll}1 & 0.85 & 0.96\end{array}\right)$ & $\left.\begin{array}{ll}0.87 & 0.92\end{array}\right)$ \\
\hline $\begin{array}{lll}0.85 & 1 & 0.93\end{array}$ & $\begin{array}{lll}0.87 & 1 & 0.93\end{array}$ \\
\hline$\left(\begin{array}{lll}0.96 & 0.93 & 1\end{array}\right.$ & $\begin{array}{lll}0.92 & 0.93 & 1\end{array}$ \\
\hline $\mathrm{YYH}, R_{H}^{\mathrm{Tx}}$ & $\mathrm{YYH}, R_{H}^{\mathrm{Rx}}$ \\
\hline$\left(\begin{array}{lll}1 & 0.21 & 0.82\end{array}\right)$ & $\left(\begin{array}{lll}1 & 0.29 & 0.86\end{array}\right)$ \\
\hline $\begin{array}{lll}0.21 & 1 & 0.28\end{array}$ & $\begin{array}{lll}0.29 & 1 & 0.32\end{array}$ \\
\hline $\begin{array}{lll}0.82 & 0.28 & 1\end{array}$ & $\left.\begin{array}{lll}0.86 & 0.32 & 1\end{array}\right)$ \\
\hline $\mathrm{VHH}, R_{H}^{\mathrm{Tx}}$ & VHH, $R_{H}^{\mathrm{Rx}}$ \\
\hline
\end{tabular}

We now present results from the analysis of the measurement data in CDFs. The channel capacities of the four polarization schemes obtained from measurement and simulation analyses are, respectively, shown as straight and dotted lines in Figure 8 and Figure 9. The four polarization schemes - VVV, YVY, YYH, and VHH - are indicated by the symbols, $\diamond, \nabla, \bigcirc$, and $\square$, respectively. The signal-to-noise ratio is set at $10 \mathrm{~dB}$ to plot the CDF of the capacity statistical properties. From the preliminary analysis result shown in Figure 8, we find that the YVY structure has the best capacity among all the investigated antenna array configurations. Moreover, when the normalized power matrix is considered with the analysis of channel capacity, the VHH scheme has the worst channel capacity among the polarization combinations. 
TABLE 6: Normalized power for different polarization schemes in indoor LOS environment.

\begin{tabular}{cc}
$\left(\begin{array}{lll}1.28 & 0.98 & 1.02 \\
1.07 & 1.23 & 1.19 \\
0.69 & 0.67 & 0.76\end{array}\right)$ & $\left(\begin{array}{lll}1.03 & 0.70 & 0.30 \\
0.66 & 1.3 & 60.61 \\
0.22 & 0.74 & 1.01\end{array}\right)$ \\
VVV & YVY \\
$\left(\begin{array}{lll}0.75 & 0.26 & 0.47 \\
0.32 & 0.97 & 0.31 \\
0.26 & 0.30 & 0.48\end{array}\right)$ & $\left(\begin{array}{lll}0.75 & 0.09 & 0.14 \\
0.10 & 0.06 & 0.11 \\
0.13 & 0.10 & 0.23\end{array}\right)$ \\
YYH & VHH \\
\hline
\end{tabular}

TABLE 7: Normalized power for different polarization schemes in indoor NLOS environment.

\begin{tabular}{cc}
$\left(\begin{array}{lll}0.80 & 1.00 & 1.09 \\
0.90 & 1.21 & 1.27 \\
0.73 & 0.96 & 1.03\end{array}\right)$ & $\left(\begin{array}{lll}1.04 & 0.55 & 0.17 \\
0.62 & 1.03 & 0.74 \\
0.14 & 0.50 & 1.11\end{array}\right)$ \\
VVV & $\left.\begin{array}{ccc}\text { YVY } \\
0.96 & 0.13 & 0.61 \\
0.19 & 0.88 & 0.79 \\
0.42 & 0.55 & 1.33\end{array}\right)$ \\
YYH & $\left(\begin{array}{cccc}1.00 & 0.10 & 0.12 \\
0.13 & 0.12 & 0.16 \\
0.12 & 0.05 & 0.61\end{array}\right)$ \\
\hline
\end{tabular}

By observing the CDF of capacity analysis under different conditions, it is found that the simulation analysis method, which makes use of (15), is close to a realistic measurement environment. This method is useful for application to polarization schemes. As seen in Figure 8 , using the YVY scheme in an indoor environment provides higher channel capacity owing to antenna isolation and greater total received power. As a result, we need to consider both isolation and correlation properties for describing an MIMO system that utilizes orthogonal polarized antennas. Using only one property is insufficient in determining the system performance. Therefore, the correlation property is not a sufficient measure when considering parallel transmission with orthogonal polarized schemes.

For the NLOS condition, as shown in Figure 9, we find that the YYH scheme has a higher capacity than the YVY scheme. As for the analysis result in Figure 7, $\lambda_{1}$ has the same power level for both the YYH and YVY schemes, but the other eigenvalues in the YYH scheme are closer to each other. Therefore, we have verified our proposed simulation analysis method and found it suitable for approximating polarization transmission and reception systems. Consequently, in attaining higher channel capacity in MIMO systems, there is a tradeoff between antenna isolation and total received power in each transceiver structure.

\section{Conclusion}

We introduced the concept of the MIMO system and factors that determined the channel capacity, including eigenvalues and correlation coefficients. We studied the effect of space and polarization diversity on the MIMO system in detail. Furthermore, we designed a series of experiments
TABLE 8: Average normalized power for various polarization schemes.

\begin{tabular}{lcccc}
\hline \multirow{2}{*}{ Measurement types } & \multicolumn{4}{c}{ Polarization schemes } \\
& VVV & YVY & YYH & VHH \\
\hline LOS & 1 & 0.73 & 0.46 & 0.19 \\
NLOS & 1 & 0.66 & 0.65 & 0.27 \\
\hline
\end{tabular}

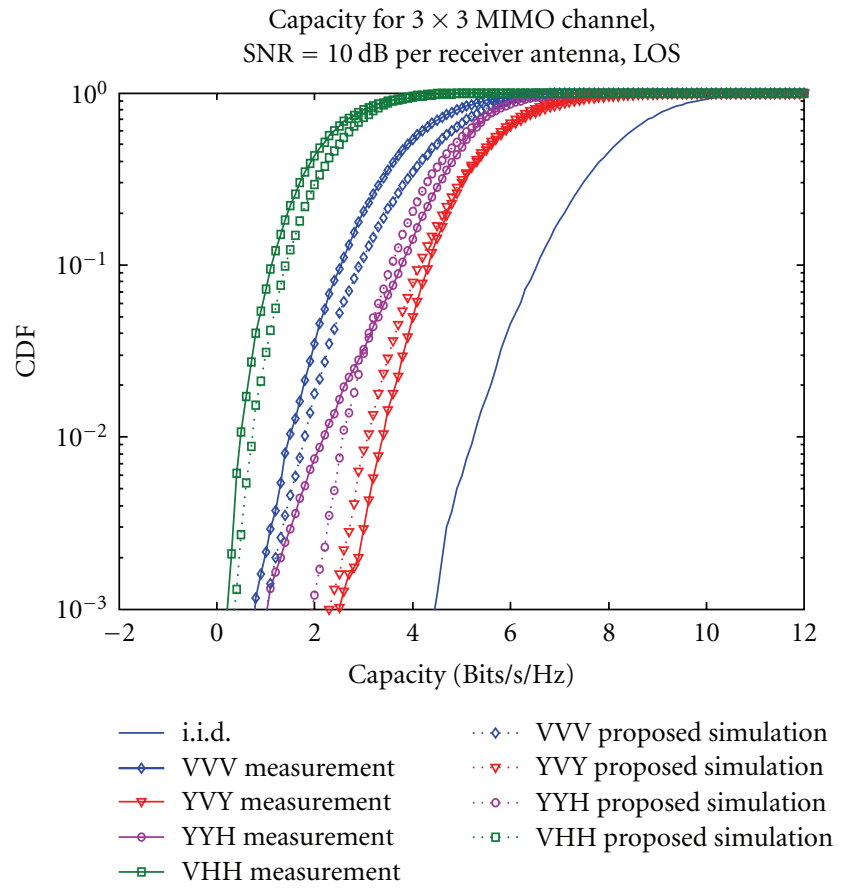

Figure 8: The plots of CDF versus capacities obtained by measurement analysis (solid line), proposed simulation analysis (dotted line) for dissimilar polarization systems in indoor LOS environment.

to determine the correlation between antennapolarization and the channel characteristics. From measurement data, the channel capacity and correlation coefficient were used for explaining the effects of various polarization schemes in the MIMO channels. Moreover, the concepts of normalizing the received power and the polarization effect were described in modifying the numerical analysis of the polarized channel capacity. In addition, we found that the performance of an MIMO system exploiting a copolarized antenna combination can be described simply using spatial correlation properties, but when adopting a cross-polarized antenna combination, both the isolation and correlation properties were needed to fully describe the system performance. Consequently, we found and verified the proposed algorithm of (15) which was close to realistic environment in polarization systems. 


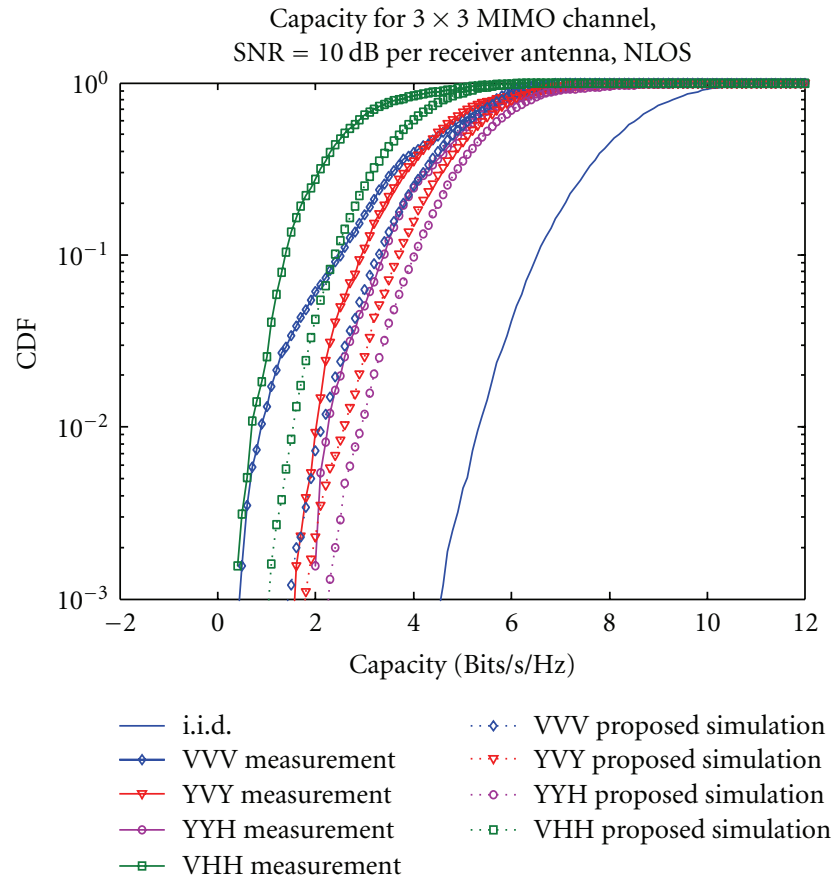

Figure 9: The plots of CDF versus capacities obtained by measurement analysis (solid line), proposed simulation analysis (dotted line) for dissimilar polarization systems in indoor NLOS environment.

\section{Acknowledgment}

This work was supported by the National Science Council, Taiwan, under the Grant of NSC95-2219-E-002-003 and NSC97-2218-E027-007.

\section{References}

[1] G. J. Foschini, "Layered space-time architecture for wireless communication in a fading environment when using multielement antennas," Bell Labs Technical Journal, vol. 1, no. 2, pp. 41-59, 1996.

[2] K. Yu, M. Bengtsson, B. Ottersten, D. McNamara, P. Karlsson, and M. Beach, "Modeling of wide-band MIMO radio channels based on NLoS indoor measurements," IEEE Transactions on Vehicular Technology, vol. 53, no. 3, pp. 655-665, 2004.

[3] L. Dong, H. Choo, R. W. Heath, and H. Ling, "Simulation of MIMO channel capacity with antenna polarization diversity," IEEE Transactions on Wireless Communications, vol. 4, no. 4, pp. 1869-1872, 2005.

[4] J. P. Kermoal, L. Schumacher, K. I. Pedersen, P. E. Mogensen, and F. Frederiksen, "A stochastic MIMO radio channel model with experimental validation," IEEE Journal on Selected Areas in Communications, vol. 20, no. 6, pp. 1211-1226, 2002.

[5] D.-S. Shiu, G. J. Foschini, M. J. Gans, and J. M. Kahn, "Fading corre- lation and its effect on the capacity of multielement antenna system," IEEE Transactions on Communications, vol. 48, pp. 502-513, 2000.

[6] J. P. Kermoal et al., "Experimental investigation of the joint spatial and polarisation diversity for MIMO radio channel," in Proceedings of the 4th International Symposium on Wireless Personal Multimedia Communication (WPMC '01), pp. 147152, September 2001.

[7] P. Kyritsi, D. C. Cox, R. A. Valenzuela, and P. W. Wolniansky, "Effect of antenna polarization on the capacity of a multiple element system in an indoor environment," IEEE Journal on Selected Areas in Communications, vol. 20, no. 6, pp. 12271239, 2002.

[8] R. U. Nabar, H. Bölcskei, V. Erceg, D. Gesbert, and A. J. Paulraj, "Performance of multiantenna signaling techniques in the presence of polarization diversity," IEEE Transactions on Signal Processing, vol. 50, no. 10, pp. 2553-2562, 2002.

[9] M. R. Andrews, P. P. Mitra, and R. Decarvalho, "Tripling the capacity of wireless communications using electromagnetic polarization," Nature, vol. 409, no. 6818, pp. 316-318, 2001.

[10] H. J. Li and C. H. Yu, "Correlation properties and capacity of antenna polarization combinations for MIMO radio channel," in Proceedings of the IEEE International Antennas and Propagation Symposium, pp. 503-506, San Antonio, Tex, USA, June 2003.

[11] T. Svantesson, "On capacity and correlation of multi-antenna systems employing multiple polarizations," in Proceedings of the IEEE Antennas and Propagation Society International Symposium, pp. 202-205, San Antonio, Tex, USA, June 2002.

[12] W. Weichselberger and H. Özcelik, "A novel stochastic MIMO channel model and its physical interpretation," in Proceedings of the 6th International Symposium on Wireless Personal Multimedia Communications (WPMC '03), Yokosuka, Japan, October 2003.

[13] A. M. Sayeed, "Deconstructing multiantenna fading channels," IEEE Transactions on Signal Processing, vol. 50, no. 10, pp. 2563-2579, 2002.

[14] S. R. Saunders, Antennas and Propagation for Wireless Communication systems, John Wiley \& Sons, New York, NY, USA, 1999.

[15] V. Jungnukel,, V. Pohl, and C. von Helmolt, "Capacity of MIMO Systems with closely spaced antennas," in Proceedings of the IEEE Semiannual Vehicular Technology Conference (VTC '02), chapter 3.2., Birmingham, UK, 2002.

[16] V. R. Anreddy and M. A. Ingram, "Capacity of measured ricean and rayleigh indoor MIMO channels at $2.4 \mathrm{GHz}$ with polarization and spatial diversity," in Proceedings of the IEEE Wireless Communications and Networking Conference (WCNC '06), vol. 2, pp. 946-951, April 2006.

[17] J. P. Kermoal, L. Schumacher, P. E. Mogensen, and K. I. Pedersen, "Experimental investigation of correlation properties of MIMO radio channels for indoor picocell scenarios," in Proceedings of the 52nd Vehicular Technology Conference (VTC '00), vol. 1, pp. 14-21, September 2000.

[18] V. Erceg, H. Sampath, and S. Catreux-Erceg, "Dualpolarization versus single-polarization MIMO channel measurement results and modeling," IEEE Transactions on Wireless Communications, vol. 5, no. 1, pp. 28-33, 2006.

[19] I. E. Telatar, "Capacity of multi-antenna gaussian channels," Tech. Rep. BL0112170-950615-07TM, Office Official Pubilications of the European Communities, AT\&T Bell Labs., 1995.

[20] D. Gesbert, M. Shafi, D. S. Shiu, P. J. Smith, and A. Naguib, "From theory to practice: an overview of MIMO space-time coded wireless systems," IEEE Journal on Selected Areas in Communications, vol. 21, no. 3, pp. 281-302, 2003.

[21] T. S. Rappaport, Wireless Communications: Principles and Practices, Prentice Hall, Upper Saddle River, NJ, USA, 2nd edition, 2002. 
[22] P. Soma, D. S. Baum, V. Erceg, R. Krishnamoorthy, and A. J. Paulraj, "Analysis and modeling of Multiple-Input Multiple-Output (MIMO) radio channel based on outdoor measurements conducted at $2.5 \mathrm{GHz}$ for fixed BWA applications," in Proceeding of IEEE International Conference on Communications, pp. 272-276, May 2002. 

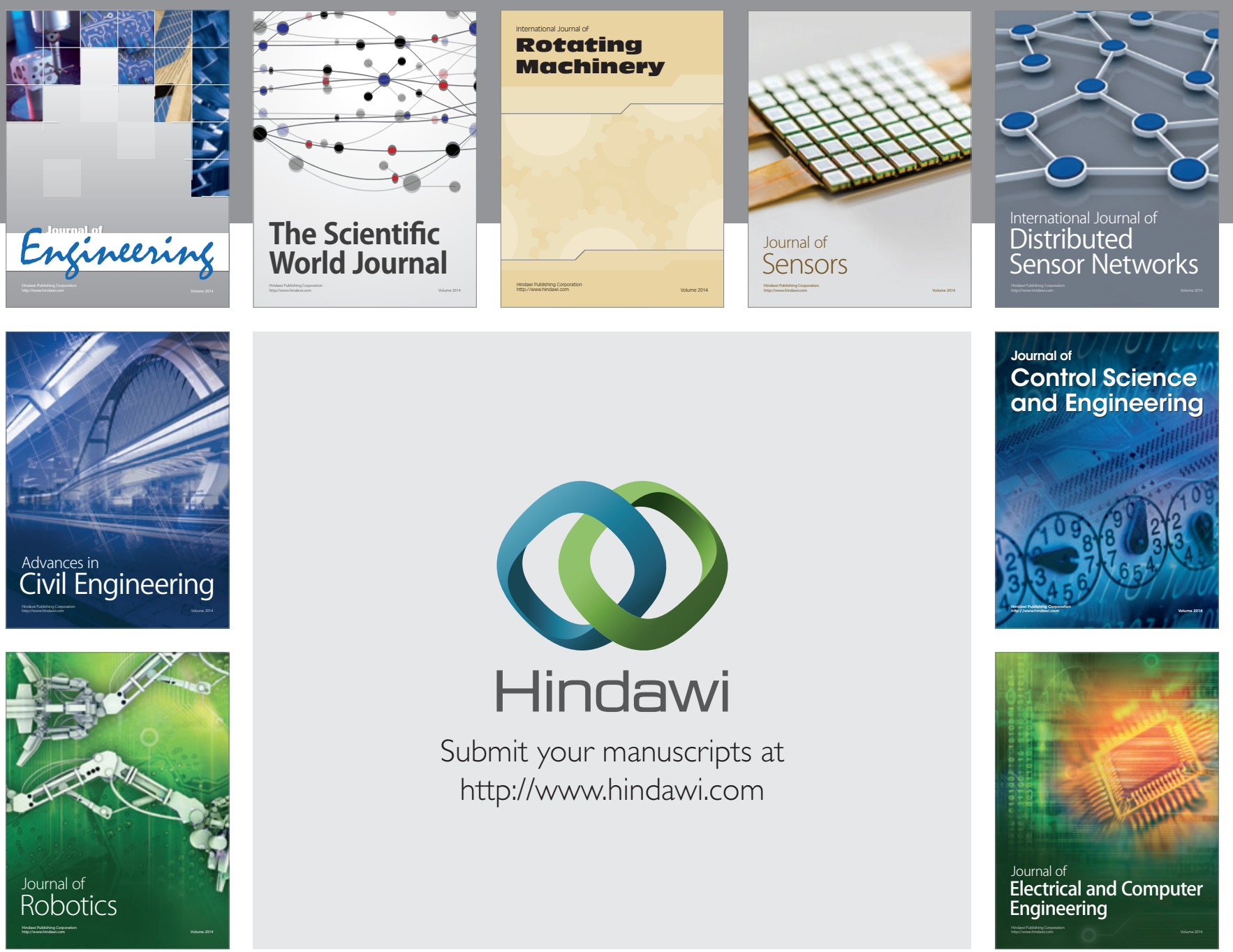

Submit your manuscripts at

http://www.hindawi.com
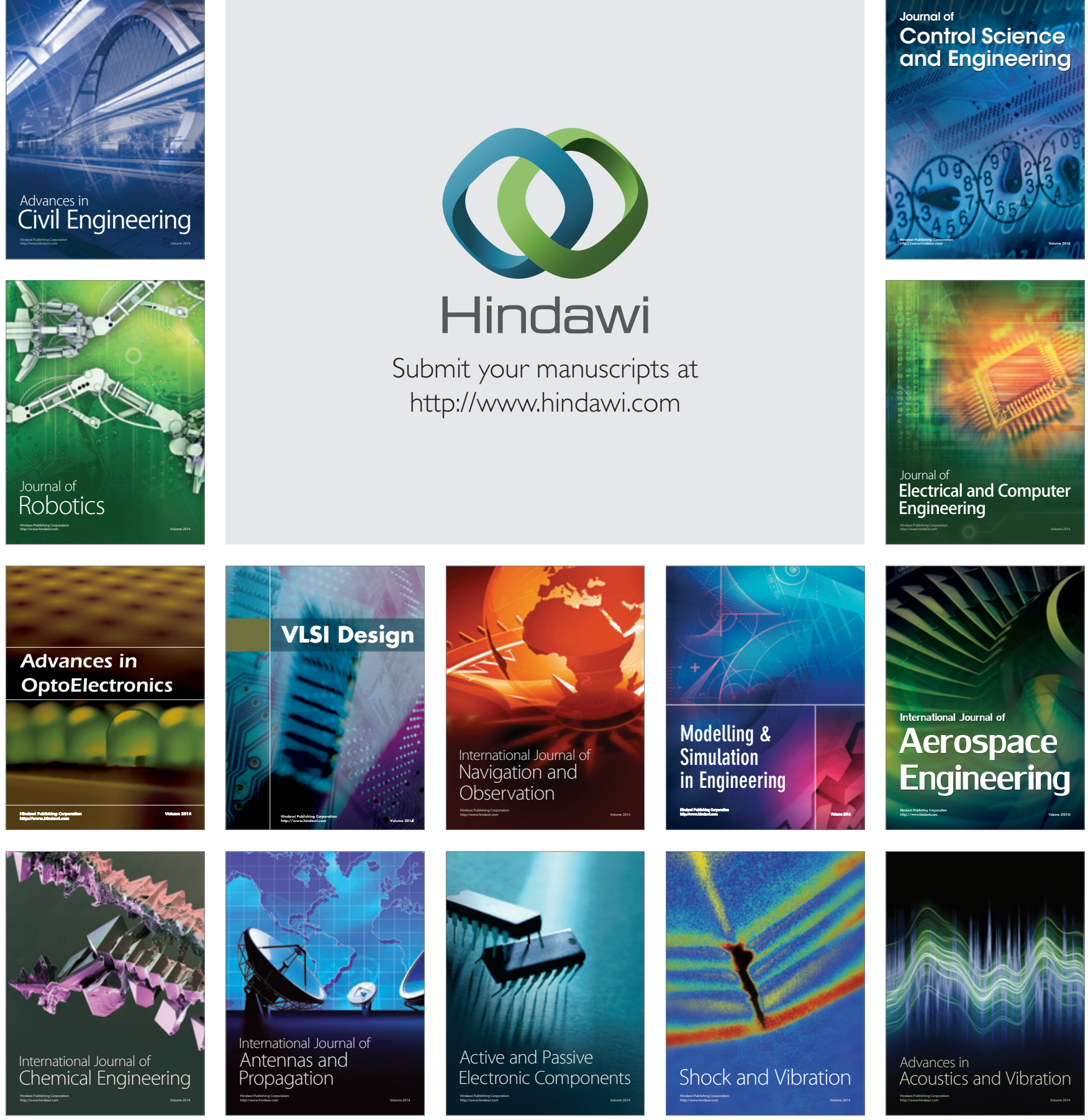